Rowan University

Rowan Digital Works

Stratford Campus Research Day

23rd Annual Research Day

May 2nd, 12:00 AM

\title{
Torsion Does Not Affect Early Vein Graft Patency in the Rat Femoral Artery Model
}

Amro Harb

Maxwell Levi

Rowan University

Akio Kozato

Robert J. Strauch MD

Follow this and additional works at: https://rdw.rowan.edu/stratford_research_day

Part of the Surgical Procedures, Operative Commons

Let us know how access to this document benefits you - share your thoughts on our feedback form.

Harb, Amro; Levi, Maxwell; Kozato, Akio; and Strauch, Robert J. MD, "Torsion Does Not Affect Early Vein Graft Patency in the Rat Femoral Artery Model" (2019). Stratford Campus Research Day. 7. https://rdw.rowan.edu/stratford_research_day/2019/may2/7

This Poster is brought to you for free and open access by the Conferences, Events, and Symposia at Rowan Digital Works. It has been accepted for inclusion in Stratford Campus Research Day by an authorized administrator of Rowan Digital Works. 


\section{Torsion Does Not Affect Garly Vein Graft Patency in the Rat Femoral Artery Model}

\section{Created by: Maxwell Levi}

Rowan University School of Osteopathic Medicine

Abstract

ackground

graft tailure in the clinical setting

The objective of this study was to determine if torsion would lead to early vein graft

Methods

25 Sprague-Dawley rats were divided into 5 equal cohorts.

degrees) (Fig. 1).

Torson wass 17. - Average vessel diameter was $1 \mathrm{~mm}$

Results

- All vein grafts were patent 2 and 24 hours post-operation.

Average blood flow rate measurements for $0,45,90,135$, and 180 degrees of torsion 2
hours post-operation: $0.37 \pm 0.02,0.38 \pm 0.04,0.34 \pm 0.01,0.33 \pm 0.01$, and $0.29 \pm 0.02$ $\mathrm{m} / \mathrm{min}$ respectively. $\mathrm{m} / \mathrm{min}$ respectively

Conclusion:

- Torsion of up to 180 degrees does not affect early vein graft patency in rat models. - Suggestion to improve clinical reproducibility of practicing vein graft procedures in rat

Introduction

Vein grafts are used to bridge vessel gaps in situations whe

- Microsurgery training courses incorporated interpositional vein graft procedures, using femoral or epigats vents as stats, in their curricula to provide surgeons wh cinically

- At the Microsurgery Training and Research Laboratory of the Columbia University Irving Medical Center, it was found that torsion of the vein graft is a common technical error made by surgeon

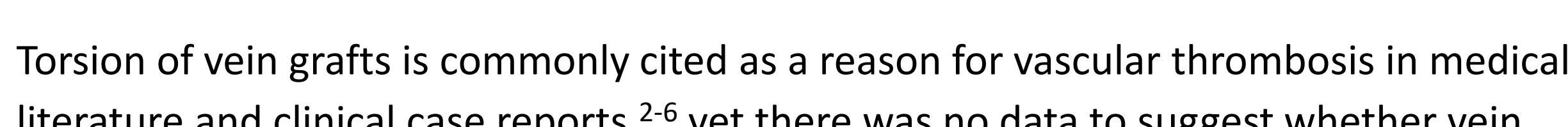
literature gaft torsion wold aed to hon,

This study aimed to determine if vein graft tors
thrombosis in non-survival surgery rat models

Methodology

Peparation

5 Sets of 5 Sprague-Dawley yats were divided into cohorts based on degree of vein graft torsion

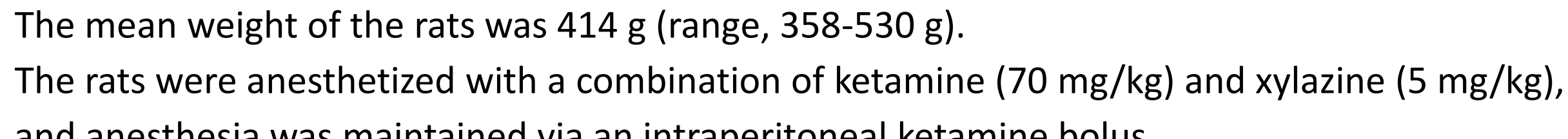

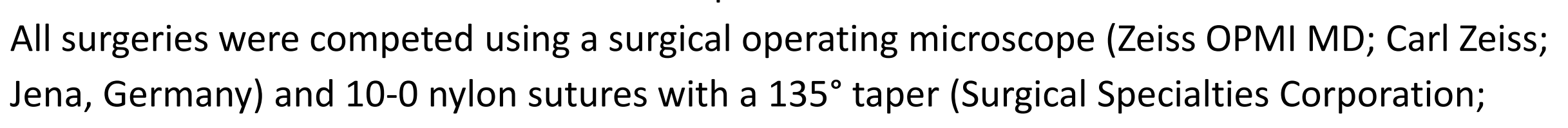

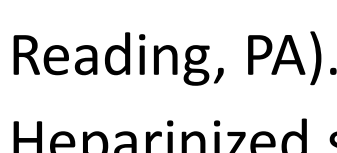

- Heparinized saline was used to irizizate the vessels throuhhout the procedure, and $1 \%$ \% Iidocaine
solution was applied to relieve any vascular spass.?

Preparing the artery

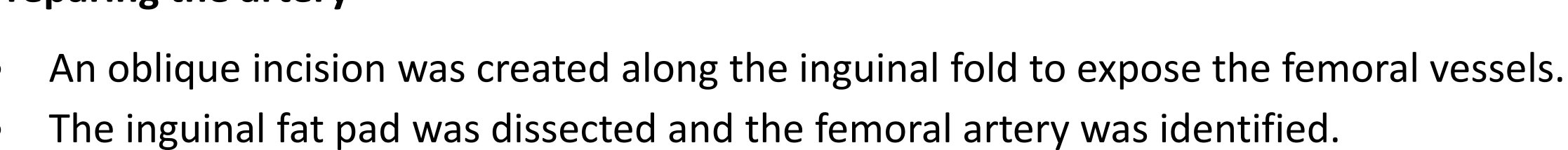

Single vascular clamps were applied to the femoral artery near the inguinal ligament tproximallt.

Adelect was created in the artery yt the midline between the clamps, and the vessel ends were
irrigated with heparinized saline, trimmed for adventitia, and dilated.

Harvesting the vein graft

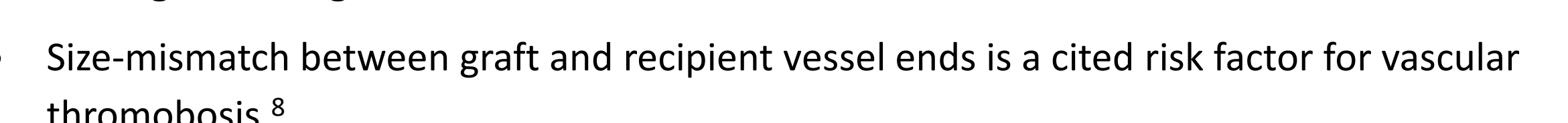

- To avoid this is ssue, the superficial epizastric vein was used for the graft as it tiameter is similar to

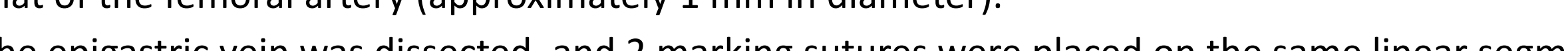

on the vevin's surface at a distance of $5.0 \pm 0.5 \mathrm{sm}$ from each other.
The

2 - Theating sutures were placed

ONear the superficial epigastric branch
O Near the inguinal fat pad (distally)
The vein graft was then harvested and

And vein grats

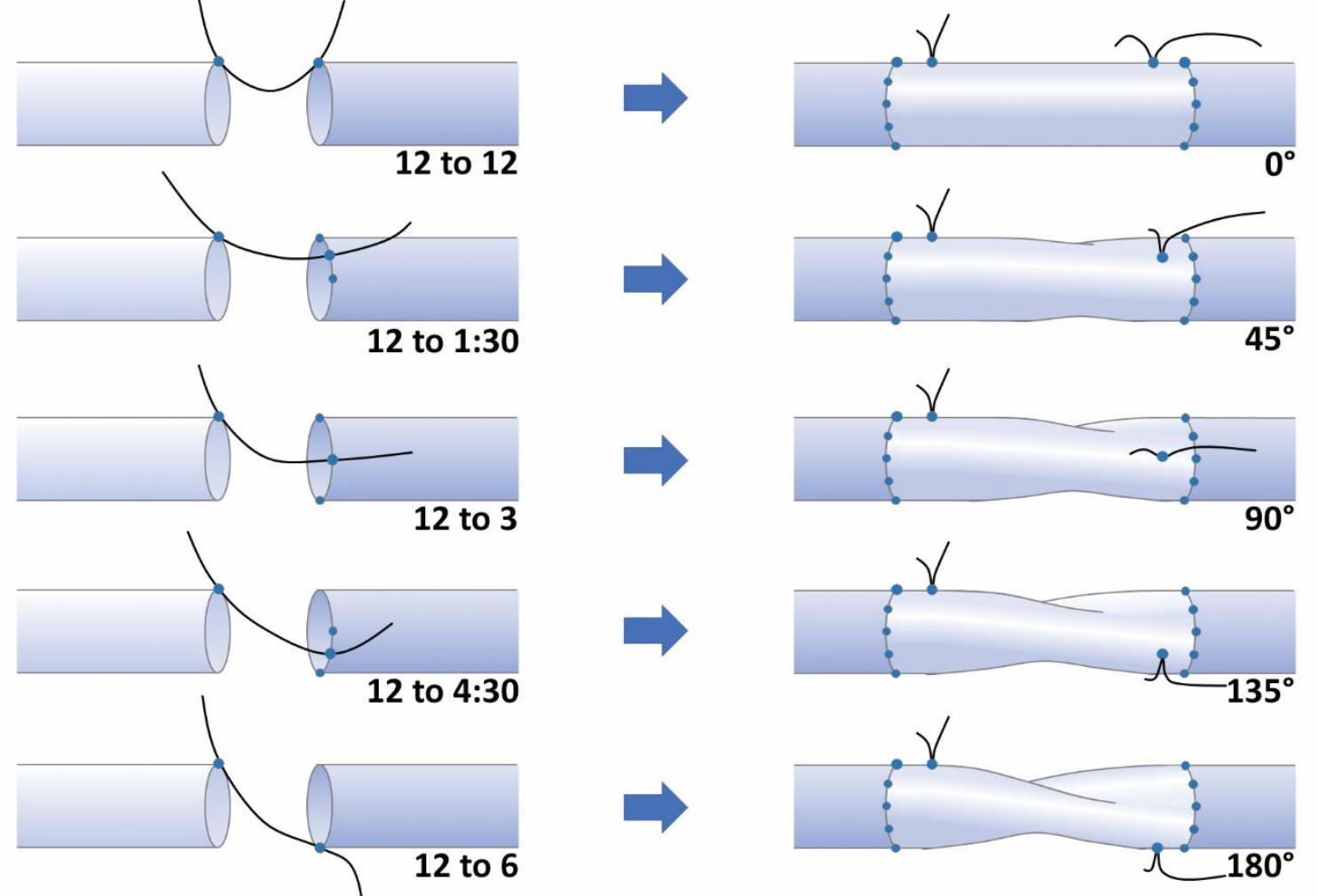

Fig 1. Left column: diagram of mismatched stitches

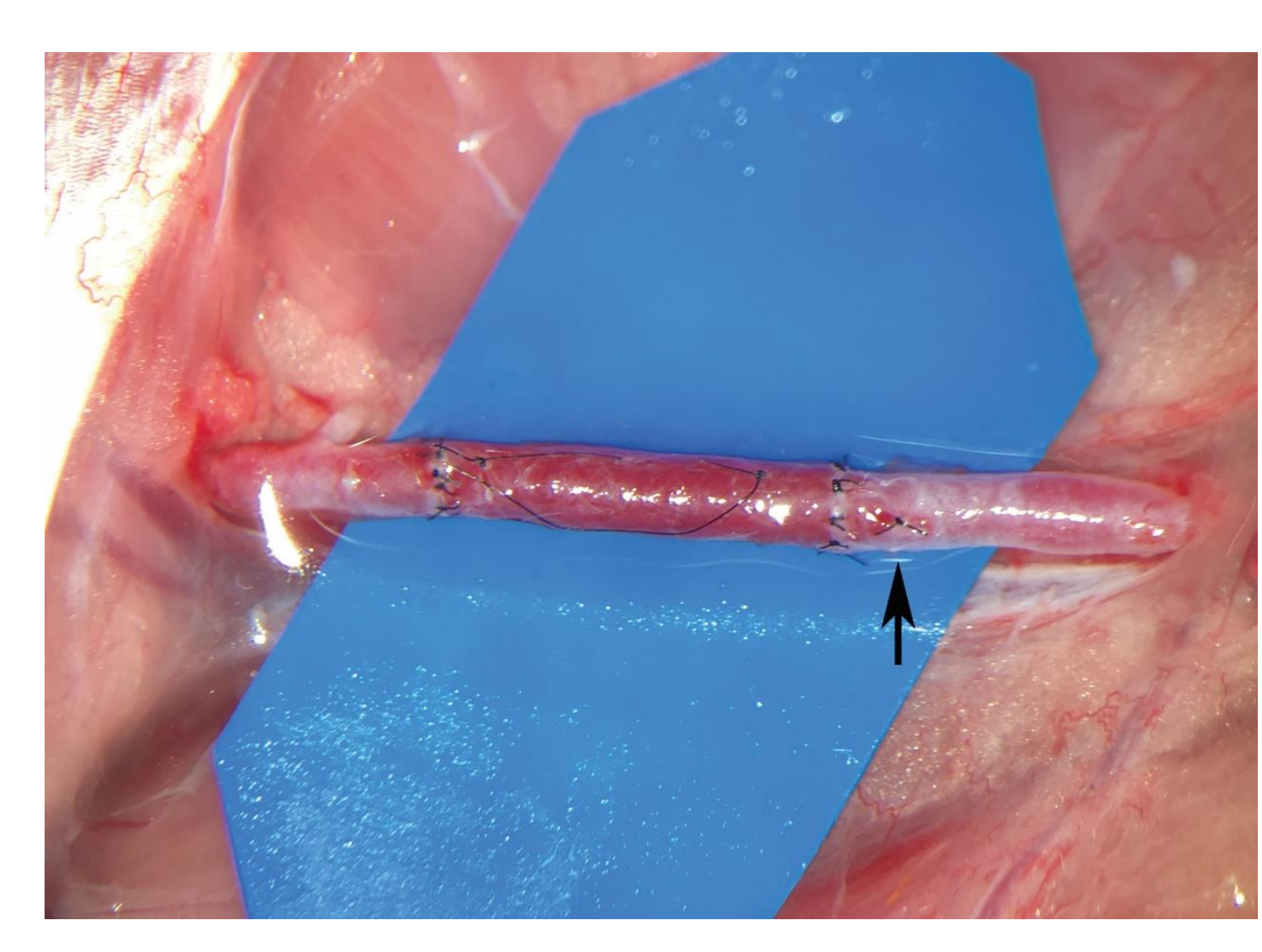

Fig 4 . Completed vein graft with 90 ded
torsion, after releasing clamps
- The anastomosis was completed with 8 circumferential sutures.i.

Stay sutures were initially placed at the 12 and 6 'llock positions.
A third suture was placed at the $30^{\prime}$ 'clock postion, and the tail of fits suture was leff long as as

handle to place the 1:30 and 4:30 o'clock sutures.

- The vessel was then rotated, and the sixht suture was placed at the $90^{\prime}$ 'lock position with its tail
left long as a handle.

The remaining
anastomosis.

e distal arterial end by mismatching sutures placed

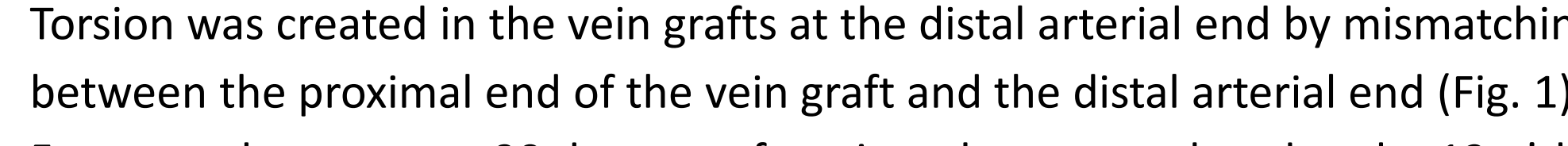

For example, to create 90 degrees of torsion, the suture placed at the 12 'clock position on the

(Fig. 21.

The next suture placed connected the $6 \sigma^{\prime}$ clock position on the proximal end of the vein graft

- The other 6 initertrusted circunferentialal sutureses would then be placed as described by Cooley to completet the anastomosis.10
Torsion was verified by noting

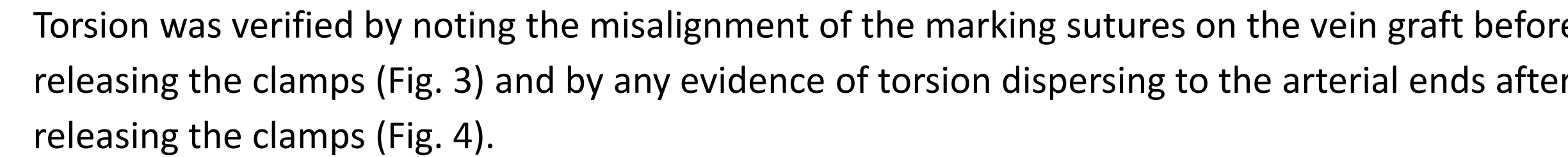

Verifying patency

- After completing both the proximal and distal anastomoses and removing the clamps, the

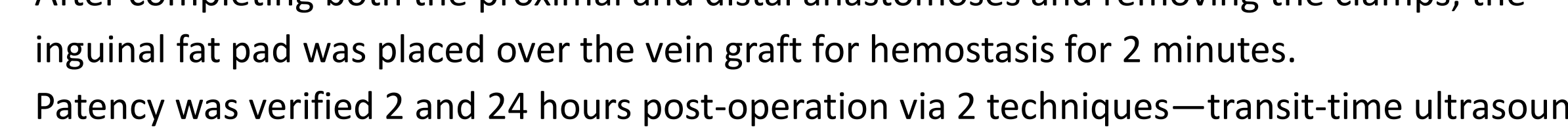
Patency was verfitied 2 and 24 hours post-operation iva 2 techniques-transit-time uitrasound
blood flow measurements and the emptr-and-refil test.11

- Blood flow measuremenents: Flow probes were placed under the distal arterial end (Fig. 5), a measurements averaging blood flow rates over 8 seconds were recorded (Fig. 6 ).
Empty-and-refil test: 2 straight edge jeweler's forceps were placed adjacent to the distal

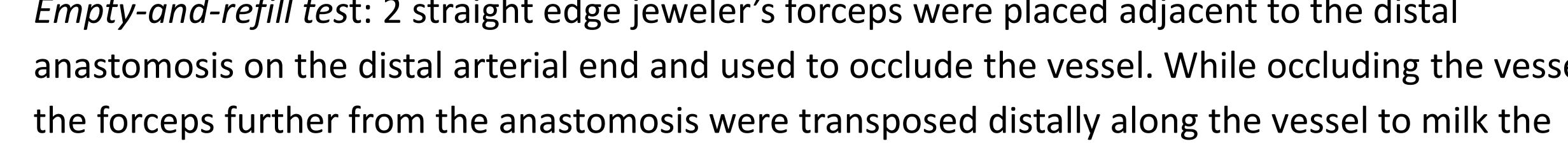

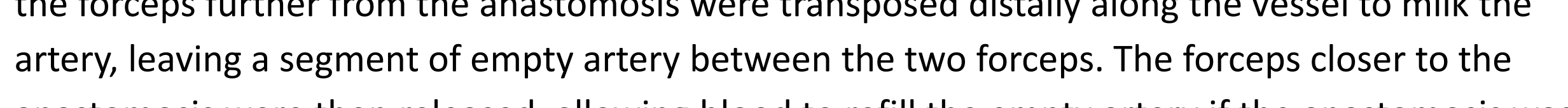
patent.

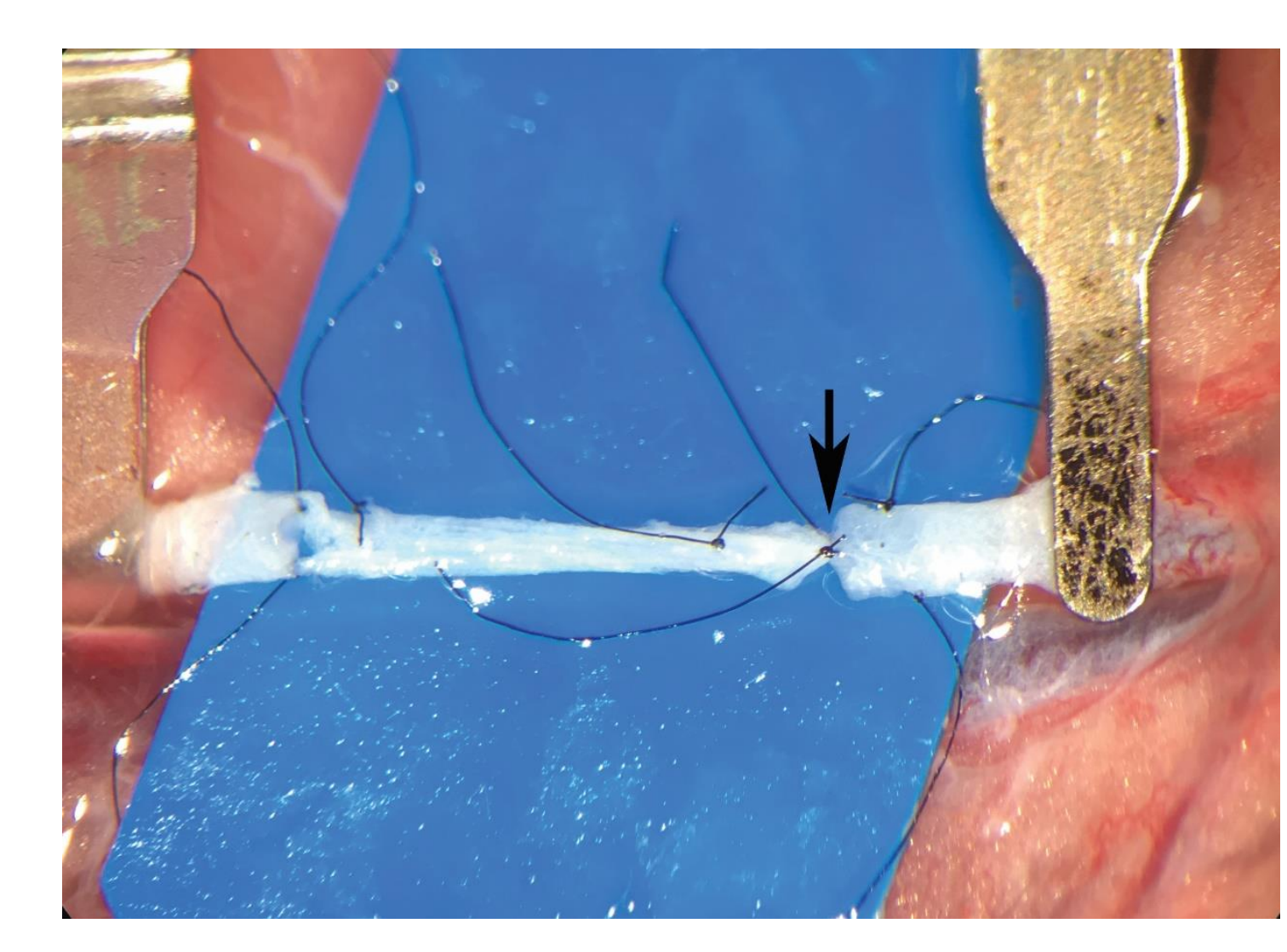

Fig 2. Vein graft with 90 degrees of torsion

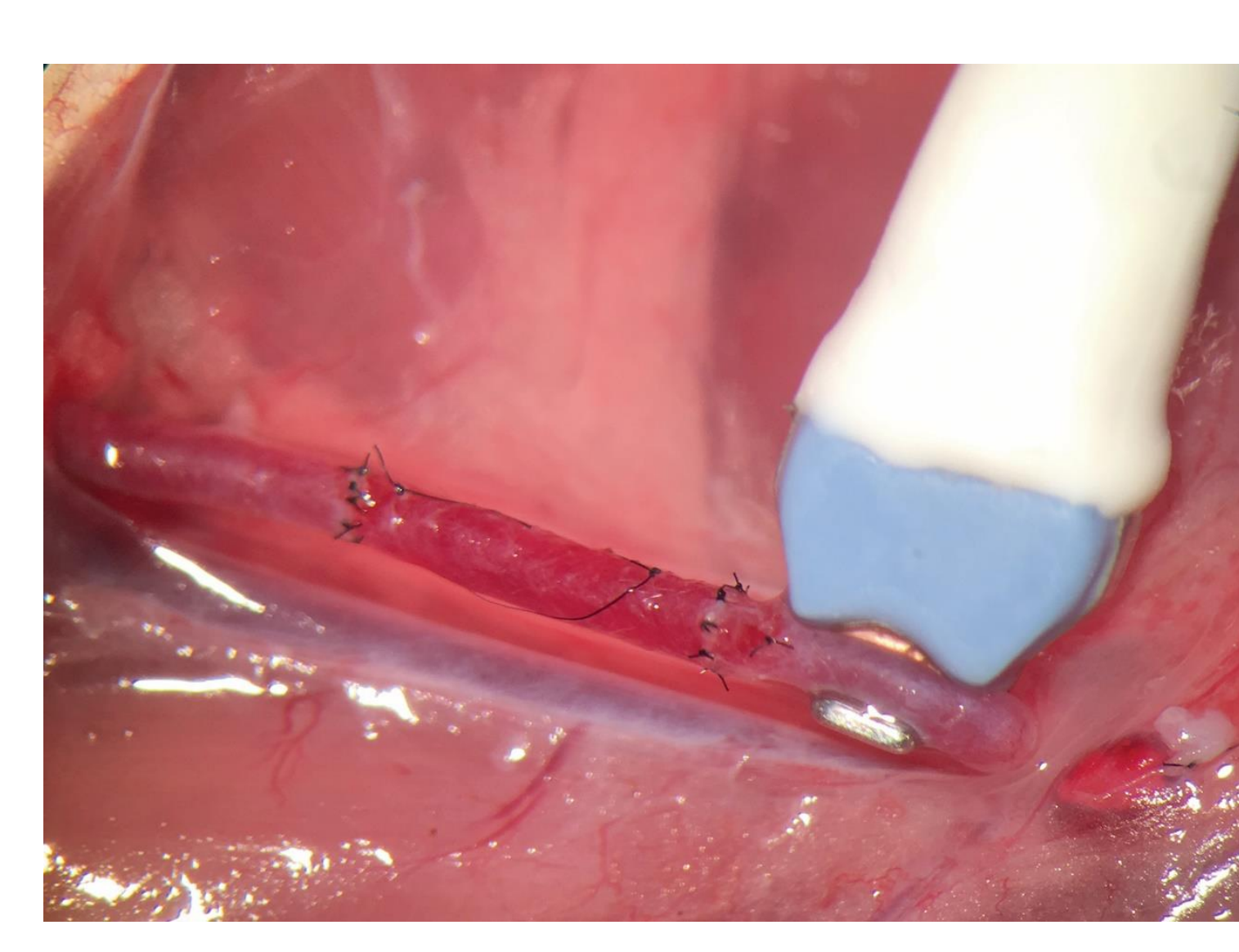

Fig 5. Verifying vein graft patency with transit-
time ultrasound blood flow measurements

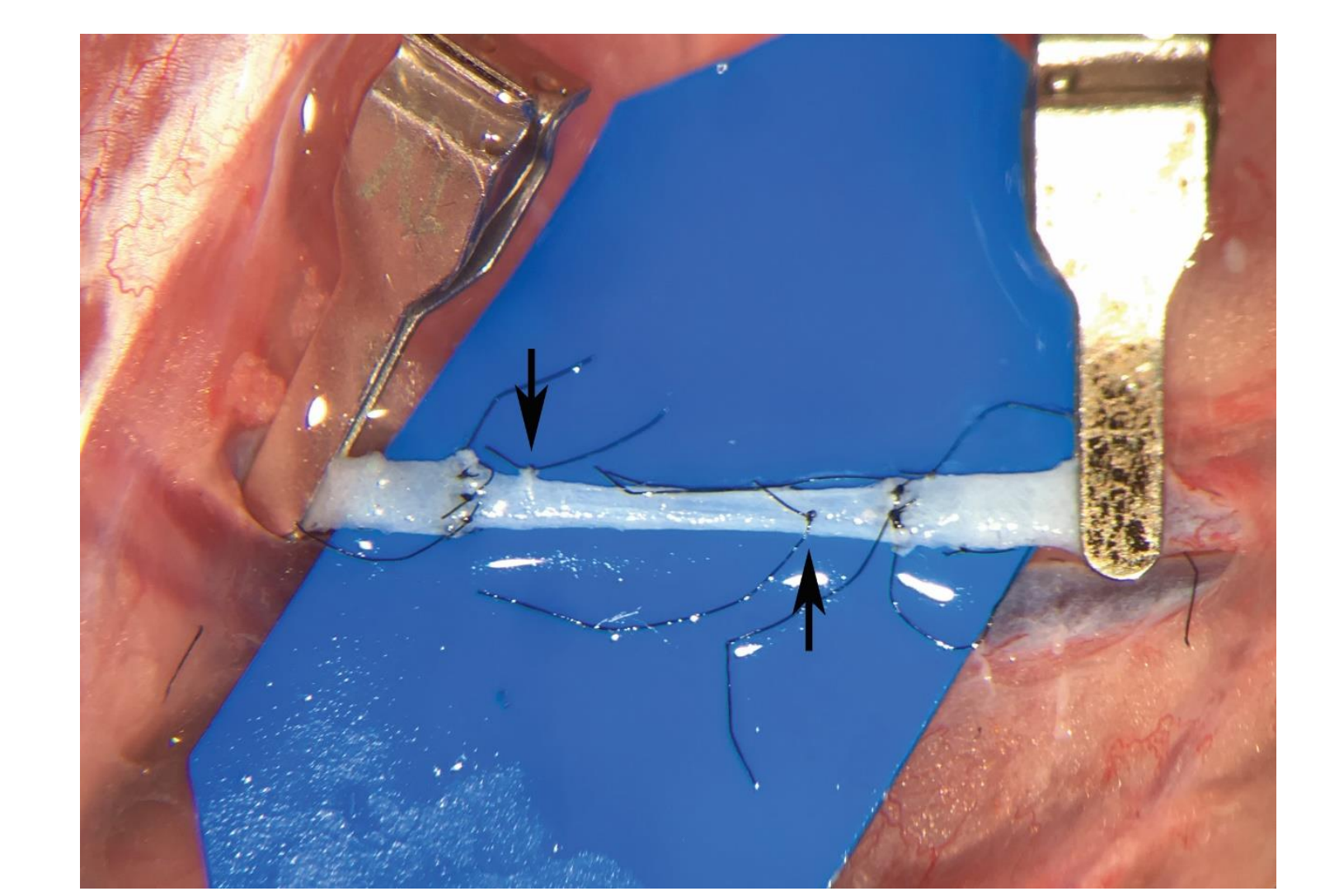

Fig 3. Completed viin graft with 90 degrees of
torsion, before releasing clamps

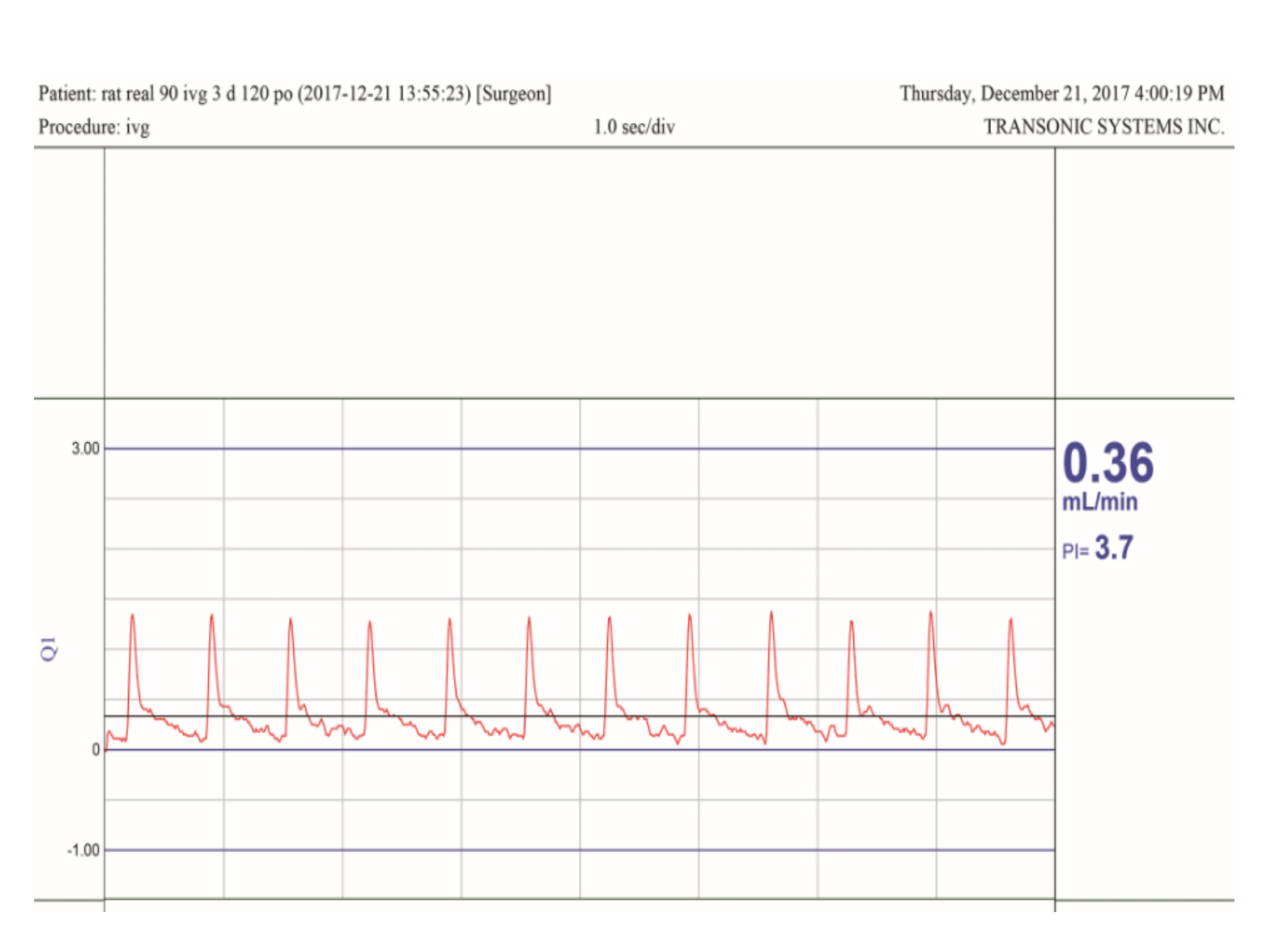

Fig 6. Blood flow rate graph recorded 2 hours
post-operation for c completed vein graft with
90 degrees of torsion

\section{Results}

All vein grafts were patent 2 and 24 hours post-operation.

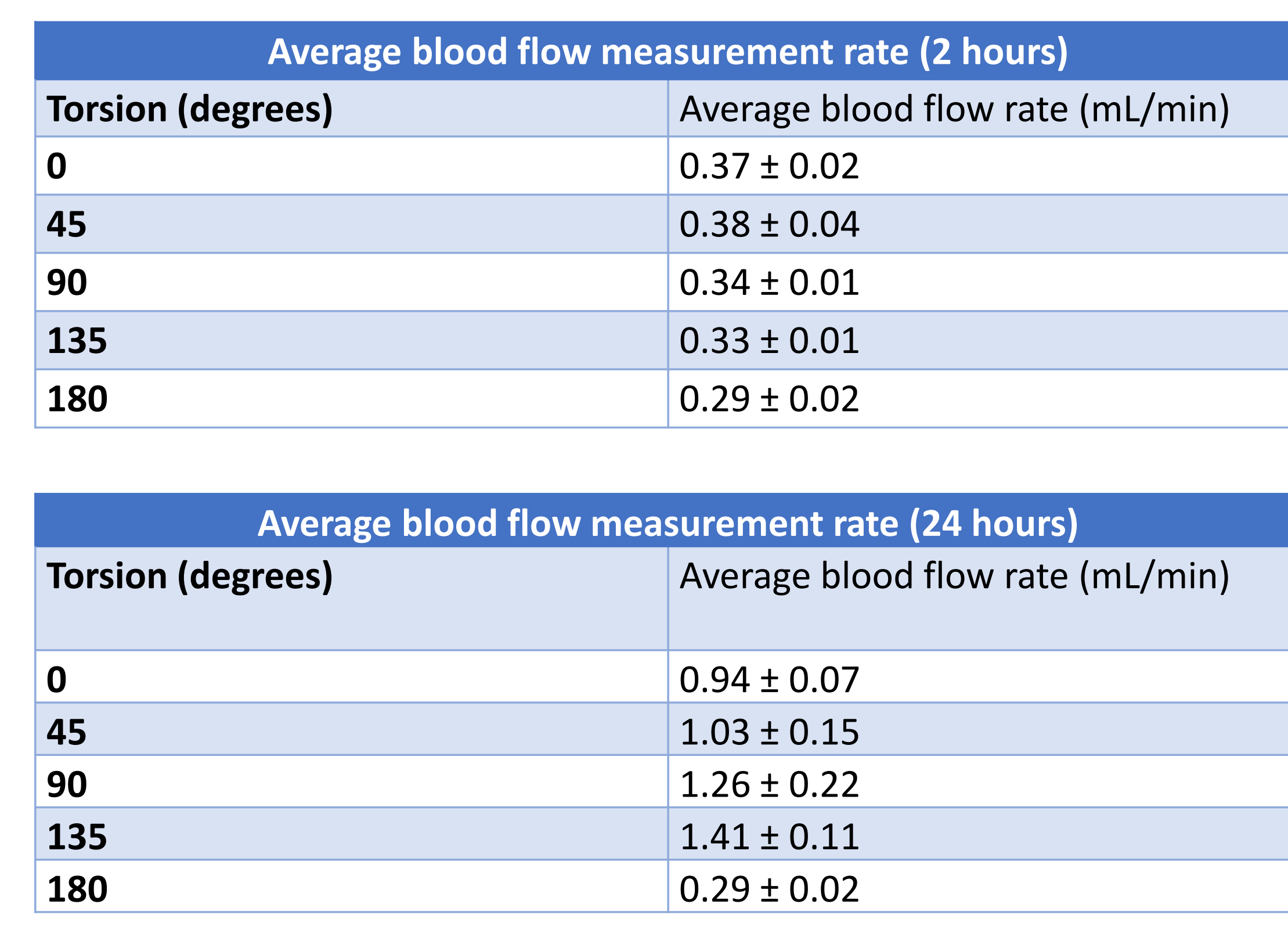

Discussion \& Conclusion

- Torsion of up to 180 degrees does not affect early vein graft patency in rat models. In human patients, it is common to minimize dissection to reduce tissue damage and
the potential of scarring, yet for interpositional vein graft exercises in rat models, the

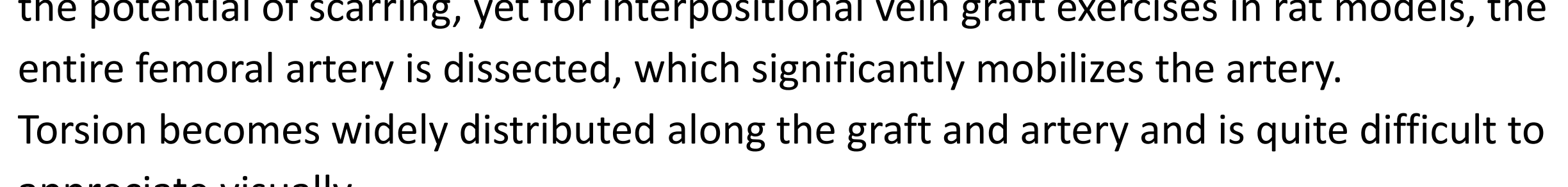
appreciate visually.

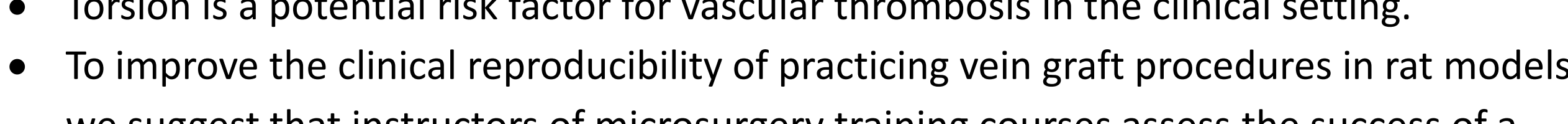
completed vein graft not only on patency but also on the basis of any torsion in the ven graft prior to clamp release.

Acknowledgements

his study was funded by an Orthopedic scientich hesearch Foundation grant to the University Irving Medical

Authors: Amro Harb, Maxwell Levi, Akio Kozato, Robert I. Strauch, MD

References

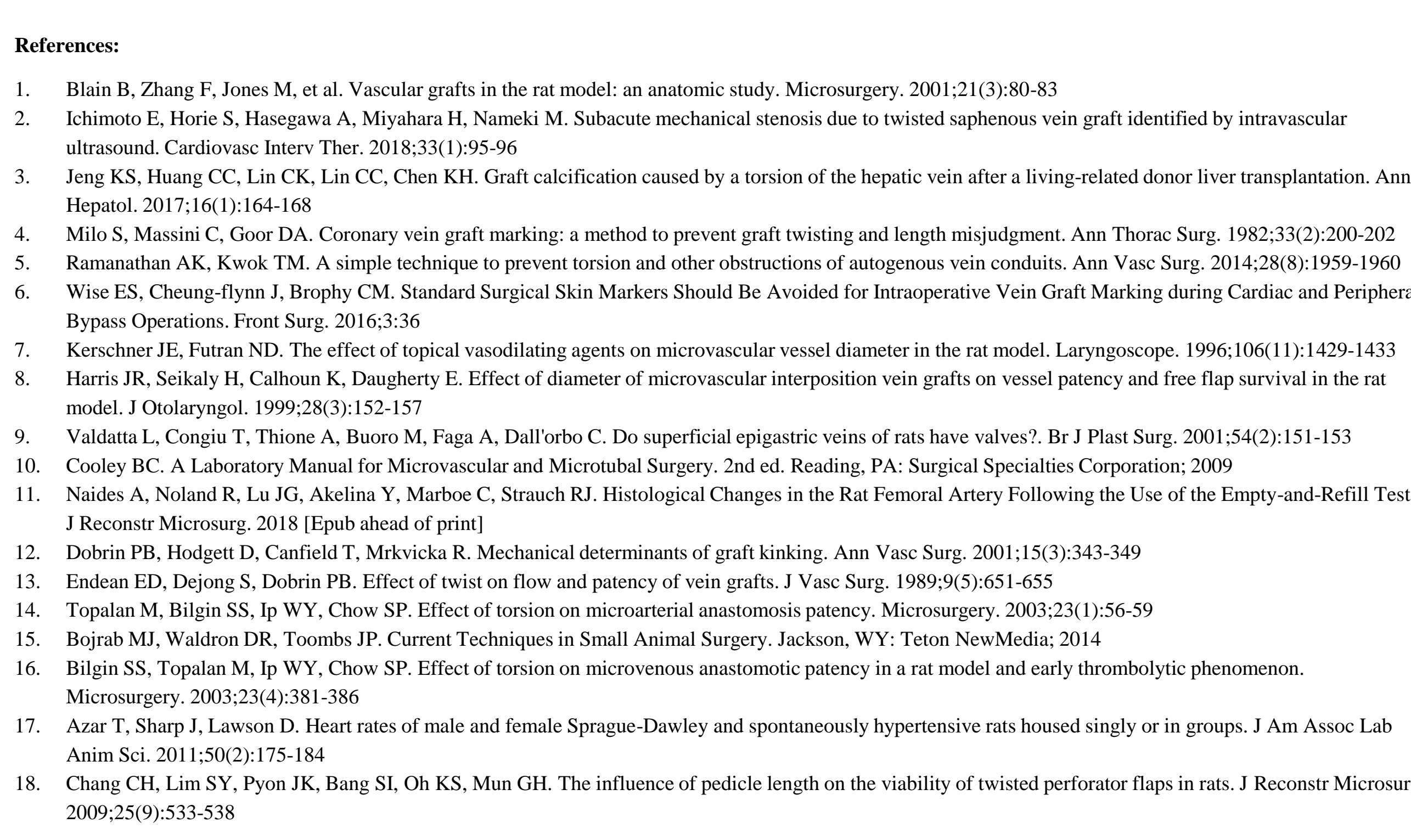

